\title{
Barocke und klassizistische Elemente in der anatomischen Abbildung
}

\author{
Von Hans-Konrad Schmutz
}

Die Anatomie des 17. Jahrhunderts trug viele barocke Züge. Bestimmt von naivneugieriger Schaulust und Sammelleidenschaft, interessierte man sich mehr für das Kuriose und Seltsame als für das Typische. Die Naturalienkabinette waren angefüllt mit Präparaten und Schaustücken, die eher die Phantasie beflügelten als wirklich informativ wirkten ${ }^{1}$.

Dieser Geisteshaltung entsprechen auch zahlreiche Darstellungen anatomischer Arbeitsstätten, so die lavierte Federzeichnung von Willem Buytewech (1585 bis 1624), die den Unterricht im anatomischen Theater zu Leiden zeigt ${ }^{2}$.

Nicht von ungefähr spielt diese Szene im holländischen Leiden. Im 17. Jahrhundert erlebten die bürgerlich regierten Niederlande ihre Blütezeit. Innert kurzer Zeit wurde das kleine Land führend in Schiffahrt, Handel, Kunst und Wissenschaft. Innerhalb der Medizin war es besonders für seine Anatomen bekannt. Dies zeigt sich an den spezifisch holländischen Anatomenbildern namhafter Künstler. Als bekanntes Beispiel sei die «Anatomie des Dr. Tulp» erwähnt, die kein Geringerer als Rembrandt festhielt ${ }^{3}$.

Zu den hervorragendsten dieser Anatomen zählt Frederik Ruysch (1638-1731). In seinen Publikationen finden sich typische Beispiele barocker Gebrauchsgraphik. Viele seiner Bücher, wie Opera anatomico-medico-chirurgica (1737) und Thesaurus anatomicus (1701-1716), sind mit Abbildungen seiner Sammlungsstücke ausgestattet.

Unter Ruyschs Hand erwachen diese Präparate zu neuem, gespensterhaftem Leben. Gezeigt wird nicht nur ein Studienobjekt, sondern Ausgüsse und Präparate werden zu surrealistisch anmutenden Landschaften zusammengestellt. Vor diesem Hintergrund agieren nun weinende Skelette, die ihrem verlorenen Leben nachtrauern. Hierzu ein Beispiel aus dem Werk «Thesaurus» (Abb. 1). Nieren- und Blasensteine bilden eine gebirgige Landschaft, bewachsen mit Bäumen und Sträuchern aus Korrosionspräparaten ${ }^{4}$. In dieser bizarren Landschaft stehen zwei etwa vier Monate alte Föten, denen folgende Losungen beigegeben sind:

- «Der Mensch, vom Weib geboren, lebt nur kurze Zeit und ist voll Gebrechen.»

- «Der Tod verschont selbst die wehrlose Jugend nicht.»

Wen wundert es, daß der am rechten Bildrand stehende Fötus ob solcher Trauer zu weinen beginnt und sich die imaginären Tränen mit einem Stück Omentum 


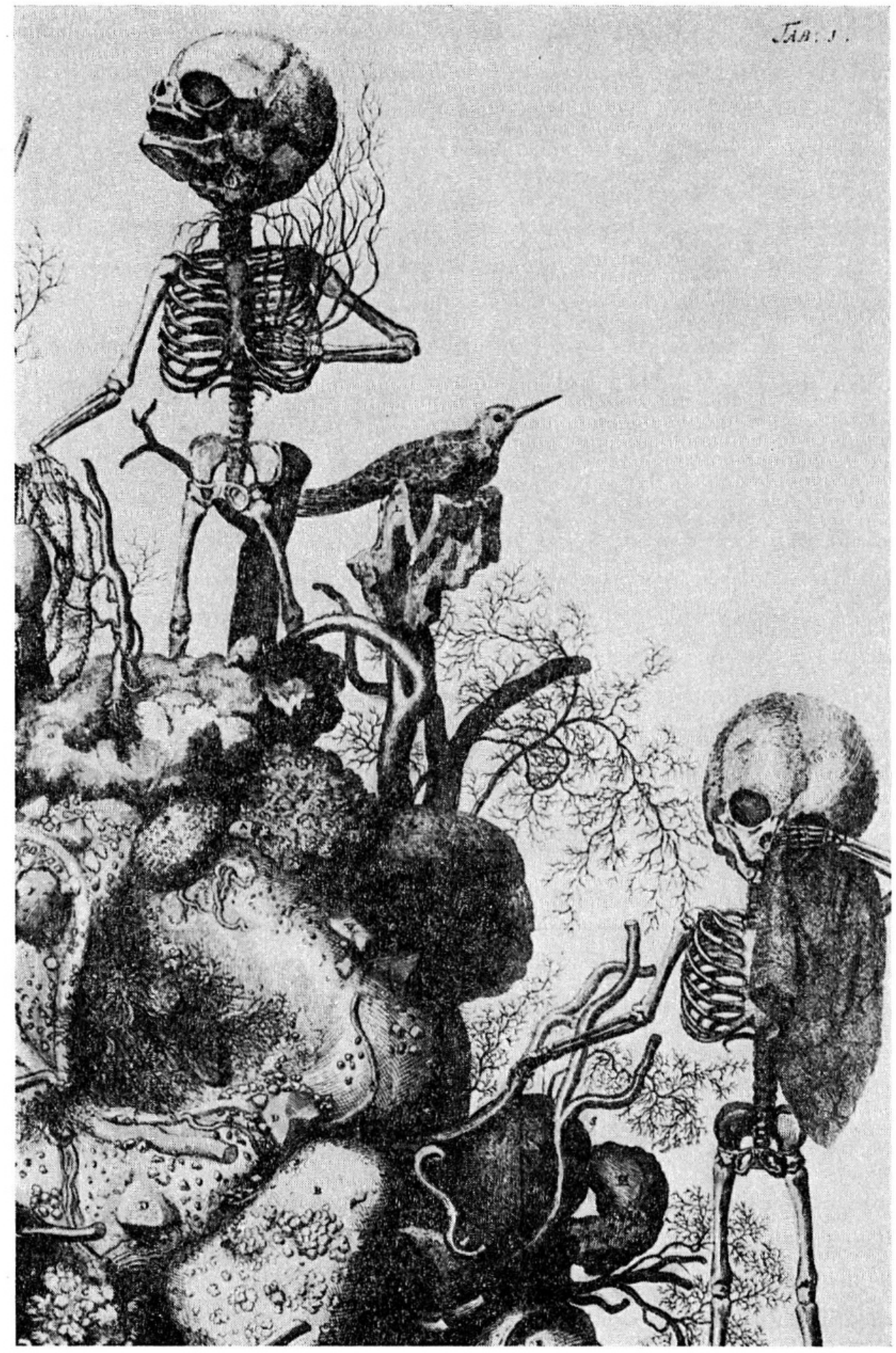

Abb. 1. Frederik Ruysch, Thesaurus anatomicus, Amsterdam 1701-1716, Thes. I, Tab. 1 (Ausschnitt). Kupferstich: $37,5 \times 33 \mathrm{~cm}$. Zeichner und Stecher : Cornelius Huyberts (1669/70-1712?) 
wegwischt? In diesen Darstellungen ist der moralische Beigeschmack unverkennbar. Fast erinnern solche Szenerien an Landschaftsvisionen eines Max Ernst oder gar eines Salvador Dali. Elemente einer manieristischen und barocken Ästhetik ergänzen sich zu einem schön-schauerlichen Spektakel, das mehr vorführen als wirklich erklären will.

Ruysch, der in seiner Freizeit selbst gemalt haben soll und dessen Töchter Anna und Rachel bekannte Blumenmalerinnen wurden, hat während seines langen Lebens mehrere Maler und Stecher beschäftigt ${ }^{5}$. Die Mehrzahl der Tafeln, wie auch Abb.1, stammen von Cornelius Huyberts, der nicht nur zeichnete, sondern auch die Übertragung auf die Kupferplatte selber besorgte. Um 1670 in Emmerich geboren, war dieser Künstler in Holland ziemlich bekannt. Davon zeugt die Tatsache, daß er für einen der begehrtesten Maler seiner Zeit, für Gerard de Lairesse, arbeiten konnte. So stammen das Titelbild zu Grondlegginge ter Teekenkunst und Tafeln zu Het groot Schilderboek von Huyberts ${ }^{6}$.

Unter Ruyschs Anleitung entstanden auch einige Buntdrucke. Das zunehmende Interesse an topographischen Zusammenhängen, die genauere Unterscheidung verschiedener Feinstrukturen und Innenansichten erforderte neue graphische Techniken. Eng verknüpft mit der Verbreitung des Buntdruckes sind Namen wie Leblon, Gauthier d'Agoty und Ladmiral.

Jean Ladmiral (1698-1773) hat unter anderem auch für Ruysch gearbeitet. Er verwendete eine Mischtechnik, indem er Schabkunst und Radierung kombinierte. Gedruckt wurde auf drei Platten, die in den Farben Rot, Gelb und Blau eingefärbt waren. Den handkolorierten Kupferstich vermochte dieses Verfahren aber nicht zu ersetzen. Dazu waren die Herstellungskosten zu hoch und oft genug die erzielte Druckqualität zu schlecht. Eine wesentliche Verbesserung brachte erst die Lithographie, die 1815 durch Martin Münz (1779-1849) in die Anatomie eingeführt wurde ${ }^{7}$.

Der erwähnte Gerard de Lairesse (1641-1711) gilt als einer der wichtigsten Vertreter des akademischen Klassizismus, d.h. jener Kunstrichtung, mit der es gelang, den holländischen Realismus gegen Ende des 17. Jahrhunderts zu überwinden. Mit der Neuentdeckung der Antike setzten sich die Künstler bewußt in einen Gegensatz zu den volkstümlichen Interieurdarstellungen ihrer Vorgänger. Der Vermittler antiker Themen war Frankreich. Wesentliche Impulse gingen von Lebrun und Poussin aus, deren Gemälde Lairesse sicher kannte. Stark gefördert wurde er durch den Kunsthändler Gerard Uilenburg, einen Verwandten Rembrandts. 1665 erwarb der geborene Lütticher das Amsterdamer Bürgerrecht. Er galt bald als bevorzugter Maler und Porträtist der Amsterdamer Kaufleute und Patrizier. Mit der völligen Erblindung fand 1689/1690 seine künstlerische Lauf- 
bahn ein jähes Ende. Den Rest seines Lebens fristete er eher kümmerlich, indem er Vorträge über theoretische Fragen zur Malerei hielt. Diese Vorlesungen wurden von seinem Sohne Abraham aufgezeichnet und in zwei Bänden herausgegeben. 1701 erschienen die Grondlegginge ter Teekenkunst und 1707 Het groot Schilderboek. Diese Bücher galten als Kompendien der Ästhetik des holländischen Klassizismus und waren, in mehrere Sprachen übersetzt, bis tief ins 18. Jahrhundert hinein von großem Einfluß. Als Graphiker beherrschte Lairesse sowohl die Technik des Kupferstiches, der Radierung als auch die der Schabkunst. Er hat die Gebrauchsgraphik und somit auch die anatomische Abbildung stark beeinflußt. Indirekt durch seine Schüler und direkt, indem er die Präparate zu Bidloos Anatomia abzeichnete.

Godefrid Bidloo gehört zu den umstrittensten Schülern von Ruysch. 1649 in Amsterdam geboren, hörte er als Lehrling eines Chirurgen Anatomievorlesungen und Demonstrationen bei Ruysch. Nach Abschluß seines Studiums diente er als Militärarzt, bis er 1688 die Nachfolge Nucks als Lektor der Anatomie und Chirurgie im Haag antreten konnte. Doch kurze Zeit später zog es ihn nach England, wo er als Leibarzt Wilhelms III. eine Anstellung fand. 1690 wurde er zum Generalsuperintendanten der Zivil- und Militärspitäler Hollands ernannt. Nach Herrlinger soll er das Jahr 1692 im Gefängnis zugebracht haben ${ }^{8}$. Sicher ist, daß Bidloo 1693 nochmals über den Kanal reiste, wo er bald als Inspektor für die Militärlazarette verantwortlich war. Ein Jahr später folgte er einem Ruf an die Universität Leiden. Jedoch unterbrach er seine Lehrtätigkeit noch mehrmals, um erneut am englischen Hofe zu dienen.

Bei seinen Zeitgenossen und auch bei manchem Historiker fand dieser ungewöhnlich bunte und stationenreiche Lebensweg wenig Beifall. So meint etwa Herrlinger: «Kein Zweifel, der Lebensweg dieses Mannes war 'barock', sein Charakter hochfahrend und eitel, er war ein Höfling und ein Ellbogenmensch.» ${ }^{9}$ Mit den Attributen «Höfling» und «Ellbogenmensch» wird man Bidloo aber bestimmt nicht gerecht.

Als erster verwendete er geschmolzenes Zinn zur Darstellung des Bronchialbaumes. 1685 schrieb er: «Die Gestalt des Bronchialbaumes läßt sich am besten darstellen, wenn man ihn durch die Trachea mit dem als Soldeertin bezeichneten geschmolzenen Zinn ausgießt. Um das Lungengewebe vom Zinn zu trennen, lege man die Lunge in Wasser und koche sie, bis der Ausguß frei daliegt, was in drei bis vier Stunden der Fall ist. ${ }^{10}$

Nebst dieser methodischen Erweiterung gelang es ihm, mit Lairesse einen der besten Zeichner seiner Zeit als Mitarbeiter zu gewinnen. Das gemeinsame Werk erschien 1685 unter dem Titel Anatomia humani corporis. Der Foliant ist, nebst 
einem allegorischen Titelblatt, mit 105 hervorragenden Kupferstichen versehen. Die Blätter enthalten keinen Hinweis auf den Namen des Stechers, zeugen aber von dessen handwerklichem Können. Die kurzen Begleittexte zu den Tafeln verfaßte Bidloo. Das großzügig gewählte Plattenformat erlaubte Lairesse, viele Präparate im Maßstab 1:1 wiederzugeben.

Die Abbildungen lassen sich in zwei Gruppen einteilen. Am Anfang stehen Ganzfiguren und Skelette. In einem zweiten Teil wird eine Anzahl Präparate demonstriert. Die Ganzfiguren erinnern nur entfernt an barocke Aktdarstellungen. Gezeichnet wurden sie vielmehr aus einem klassizistischen Stilgefühl heraus. Den Hintergrund bilden Elemente einer nachempfundenen Antike. Somit läßt sich auch die Staffage eindeutig zuordnen. Das Skelett wird nicht nur als Teil des Bewegungsapparates dargestellt, sondern ist gleichzeitig auch Symbol des Todes. Ikonographisch wichtig sind Sanduhr, Leichentuch und die Gruft, vor der die Skelette sinnend stehen.

Eigentliche Glanzstücke der Anatomia sind zweifellos die Präparatwiedergaben (Abb. 2). Der Zeichner hat versucht, die von Bidloo präparierten Stücke mit größtmöglicher Genauigkeit festzuhalten. Kaum ein Detail, das nicht mitgezeichnet und mitgestochen worden wäre. Nadeln, Sonden, Messer und Schnüre werden benützt, um das Präparat aufzubauen. Durch Abheben aufeinanderliegender Teile gelingt es, die dritte Dimension andeutungsweise sichtbar zu machen. Das Bild gewinnt an Tiefe und Ausgewogenheit. Beherrschte Lairesse als Maler die Technik der täuschenden Oberflächenmalerei, so stand ihm als Graphiker die ganze Skala der Beleuchtungseffekte und Schattierungen zur Verfügung. In seinen beiden Lehrbüchern benützte er ein reiches Vokabular, um die verschiedenen Lichtqualitäten zu beschreiben.

Neben klassizistischen Zügen finden sich in diesen Tafeln auch barocke Stilelemente. Wie Ruysch kokettieren auch Bidloo und Lairesse mit dem Sexus ${ }^{11}$. Spielerisch erscheint etwa eine Mädchenbrust oder ein zarter Frauenarm.

Die Abbildungen, welche der Leidener Anatom Bernhard Siegfried Albinus anfertigen ließ, entstammen einer anderen Geisteshaltung. Als Nachfolger von Johannes Jacobus Rau (1668-1719) auf dem Lehrstuhl der Anatomie erwarb er sich große Verdienste um die Beschreibung des Bewegungsapparates. Seine 1747 erschienenen Skelett- und Muskeltafeln erreichten große Beliebtheit und wurden mehrmals nachgestochen ${ }^{12}$. Betrachtet man diese Kupferstiche, so ist man erstaunt über die großen stilistischen Unterschiede zu Bidloos Illustrationen. Bei Albinus wird die stillebenhafte Wiedergabe der Präparate aufgegeben. Der Mensch wird nun als höchst sinnvolle Konstruktion verstanden.

Daher bemühte man sich, möglichst mathematisch-perspektivisch genau zu 


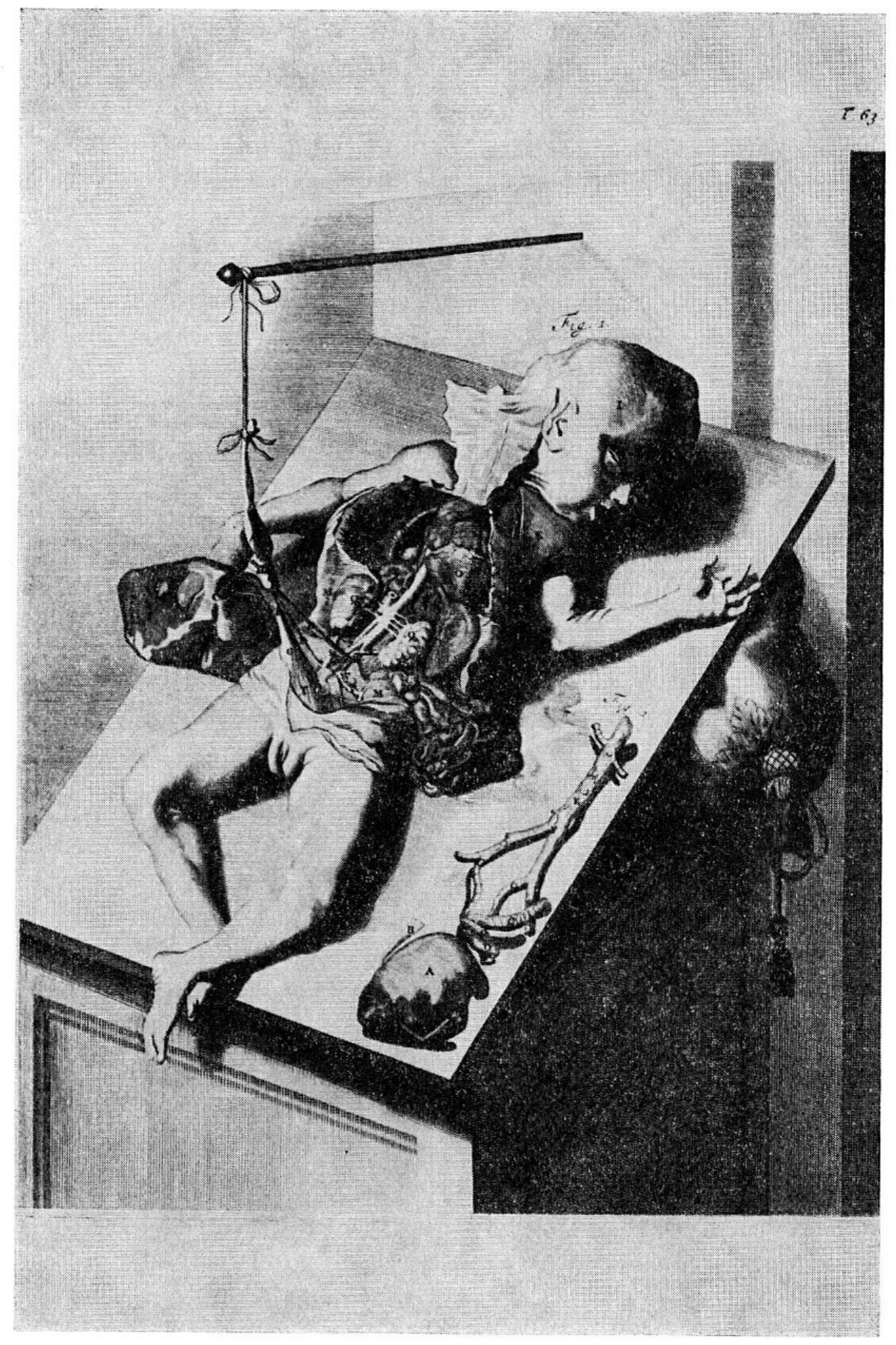

Abb. 2. Godefrid Bidloo, Anatomia humani corporis, Amsterdam 1685, Tafel 63. Kupferstich: $38 \times 53 \mathrm{~cm}$. Zeichner: Gérard de Lairesse (1640-1711). Stecher: ? Abraham Blooteling (1640-1690) 
zeichnen. Die Illustratoren bedienten sich besonderer Zeichenhilfen ${ }^{13}$. Das Präparat wurde über zwei in verschiedener Entfernung vom Objekt aufgestellte Fadennetze anvisiert und gezeichnet. Auf diesem Wege gelang es, eine perspektivisch genaue Darstellung wiederzugeben. Im 19. Jahrhundert wurde diese Zeichenhilfe weiterentwickelt und als Lucaes Zeichenapparat auf den Markt gebracht.

Albinus verzichtete noch nicht auf eine üppige Staffage; doch bleibt das Beiwerk streng auf den Hintergrund beschränkt. Es ist einem reichverzierten Theatervorhang ähnlich, vor dem die Skelette agieren. Zum Vergleich sei an Kupfertafeln aus Casserios Tabulae anatomicae erinnert, die 1627 in Venedig gedruckt wurden. Hier knien die Figuren in der Landschaft. Das Beiwerk ist in Vorder-, Mittel- und Hintergrund gegliedert und wichtig für die Komposition des Blattes.

Wie bereits erwähnt, gab Albinus 1747 seine reichillustrierten Tabulae sceleti et musculorum corporis humani heraus. Alle 40 Tafeln wurden von derselben Hand gezeichnet und gestochen. Jedes Blatt trägt die Unterschrift von Jan Wandelaar (1690-1759 $)^{14}$. Er hatte nicht nur für Ruysch und Albinus gearbeitet, sondern auch Tafeln zu Linnés Hortus Cliffortianus geliefert. Nebst Pastellarbeiten sind von Wandelaar auch Porträtstiche berühmter Anatomen wie Herman Boerhaave (1668-1738) und Arend Kant (1695-1723) erhalten geblieben ${ }^{15}$. Zeitgenossen und Historiker haben den Atlas von Albinus oft überschwänglich gelobt. So schrieb etwa Samuel Thomas Soemmerring (1755-1830): «... Um so mehr muß man die mit attischer Vollkommenheit geendigten Meisterstücke des großen Albinus zur Nachahmung empfehlen; äußerst wenige haben sie erreicht, keiner übertroffen. ${ }^{16}$

Ähnliche Ansichten über Buchillustrationen vertrat auch sein Schüler Albrecht von Haller (1708-1777). Dies zeigt sich deutlich in den zwischen 1743 und 1756 erschienenen acht Faszikeln seiner Icones anatomicae.

Das erste Heft besteht aus Einzelarbeiten zu verschiedenen Teilgebieten der Anatomie. Bereits im zweiten Faszikel wird der Plan deutlich, das Gefäßsystem zu beschreiben. Der Entschluß hierzu lag nahe, wenn man bedenkt, daß die technischen Voraussetzungen hierzu bereits im 17.Jahrhundert gelegt worden waren. Da Haller in Göttingen über ein verhältnismäßig großes Leichenmaterial verfügte, konnte er von allem Anfang an vergleichend die normale Humananatomie zur Darstellung bringen. Gesucht wurde nicht nach seltenen Varianten, wie man es Bidloo nachsagen könnte, sondern die häufigste Variante, die Norm.

Wenn man bedenkt, wie oft Venen und Arterien einen normabweichenden Verlauf zeigen können, so wird man Haller zubilligen müssen, den wohl einzig möglichen Weg zur Bearbeitung dieser Fragestellung gewählt zu haben. Er sezierte 
jedes Gefäß mitsamt dem zugehörigen Versorgungsgebiet so häufig wie möglich. Einzelne Regionen soll er bis zu 50 mal injiziert und präpariert haben, um dem Zeichner eine möglichst optimale Vorlage bieten zu können ${ }^{17}$.

Wie erwähnt, finden sich schon im zweiten Faszikel Gefäßdarstellungen (Arteria maxillaris, Arteria thyroidea sowie die Gefäßversorgung von Leber und Magen). Mit drei weiteren Heften wird diese Reihe zwischen 1745 und 1749 fortgesetzt. Die Blutversorgung der unteren Extremitäten wird 1752 publiziert; die der oberen Extremitäten und der Thorakalorgane erscheint ein Jahr später. Der VII. Faszikel enthält Tafeln zu den Themen Hirnkreislauf, Rückenmarksversorgung und eine Übersicht über die Gefäße des Auges. Mit der achten und zugleich letzten Lieferung schließt Haller das Werk ab. Dieser VIII. Faszikel zeigt zusammenfassend das Gefäßsystem des ganzen Menschen, illustriert mit zwei großen Übersichtstafeln. Haller und seinen Zeichnern war es mit den Icones gelungen, neben die Osteologie des Albinus eine gleichwertige Gefäßanatomie zu stellen. So ist Hyrtls Begeisterung verständlich; er schreibt über Haller: «Seine 'Icones' halte ich für sein größtes Werk, denn hier zeigt sich der Anatom in der Fülle seiner Gelehrsamkeit und seiner praktischen Gediegenheit. So wird denn die Dankbarkeit der Wissenschaft den Lorbeer seines Grabes auch in alle Zukunft schmücken mit immer frischem Grün, wenn von den Größen der Gegenwart und all' dem eitlen Lärm, welchen sie erregten, kein Nachhall mehr klingen wird. „ ${ }^{18}$ Ohne das Talent und den Fleiß der Zeichner und Stecher wären diese Tafeln trotz der geschickten Hand Hallers nie in dieser hohen Qualität entstanden.

Die notwendigen Zeichnungen entwarfen Joel Paul Kaltenhofer (1716-1777) und Christian Jeremias Rollin (1707 bis nach 1778). Rollin wurde als Sohn eines französischen Emigranten 1707 in Kassel geboren. Früh entschloß er sich zum Studium der Medizin. Auf einer Studienreise durch die Niederlande lernte er auch Ruyschs Technik des Einbalsamierens und der Gefäßinjektion kennen. Nach Kassel zurückgekehrt, lockte den 28jährigen der Ruf der neugegründeten Universität Göttingen. Hier hörte er auch Hallers Vorlesungen. 1742 wurde er zur Doktorprüfung zugelassen. Nun arbeitete Rollin als Prosektor und Zeichner am Anatomischen Institut. Drei Jahre später kam es aber zu heftigen Zerwürfnissen zwischen beiden ${ }^{19}$.

In der Burgerbibliothek Bern sind viele Entwürfe und Zeichnungen von Rollins Hand erhalten geblieben. Diese Studien geben einen Einblick in Technik und Strichführung des Göttinger Prosektors. Zuerst entstanden skizzenhafte Entwürfe, flächig anmutend. Die Umrisse wurden später oft mit verdünnter Tusche verdeutlicht und, wenn nötig, korrigiert. Nun übertrug Rollin diese Zeichnung auf ein neues Blatt. Durch zusätzlich angebrachte Schattierungen erhielt die 
Darstellung eine beeindruckende Plastizität. Bei der anschließenden Übertragung auf die Kupferplatte verloren die Illustrationen wenig von ihrer ursprünglichen Frische. Dies beweist die Abbildung 3. Die Darstellung zeigt die Gefäßversorgung von Magen und Leber. Bezeichnenderweise ist das Beiwerk stark reduziert. Instrumente wurden nur dann mitgezeichnet, wenn es zur anatomisch richtigen Demonstration notwendig schien.

Im Gegensatz zu Rollin war Joel Paul Kaltenhofer nicht medizinisch geschult. 1745 arbeitete er zum ersten Male für Haller. Eine feste Anstellung erhielt er allerdings erst zwei Jahre später, nachdem sich Haller und Rollin endgültig getrennt hatten. In den darauffolgenden Jahren lieferte Kaltenhofer die Vorlagen zu insgesamt 61 Kupferstichen. Einige dieser Zeichnungen hat er auch selbst auf die Kupferplatte übertragen. Allerdings lag ihm das Zeichnen weit besser als die Arbeit mit dem Grabstichel. Seine Kupfertafeln wirken leicht verwaschen, und die Tiefe der Präparate ist kaum wiedergegeben (vgl. Fasc. III, Tafel 6). Von weit besserer Qualität sind Blätter, die zwar von Kaltenhofer entworfen, aber von Georg Daniel Heumann gestochen und gedruckt wurden (vgl. Fasc. IV, Tafel 7).

Der von Albinus und Haller geförderte Stil der Buchillustration sollte bestimmend werden für die anatomische Abbildung des ausgehenden 18. und des 19. Jahrhunderts. Das Bemühen, die Schönheit menschlicher Anatomie mit Hilfe zeitgenössischer Kunst darzustellen, erlosch zunehmend. Durch die Einführung neuer Techniken wie die der Lithographie und später der Photographie wurde die anatomische Illustration zum rein didaktischen Hilfsmittel des Anatomen.

\section{Zusammenfassung}

Die anatomische Abbildung aus der Zeitspanne von Vesals Fabrica bis zur Mitte des 17. Jahrhunderts ist weitgehend von demselben Stilempfinden geprägt. Die Ganzfiguren in Casserios Tabulae anatomicae (1627) werden als lebende Wesen dargestellt. Präparate wie die aus Ruyschs Naturalienkabinett entfalten zuweilen ein groteskes Eigenleben.

Eine Stiländerung läßt sich in der zweiten Hälfte des 17.Jahrhunderts feststellen. Die Tafeln von Lairesse sind Stilleben. Naturalistisch wird das auf dem Sektionstisch liegende Präparat mit all seinen Variationen gezeichnet und gestochen. Als Gegenstand der Darstellung rückt das Präparat immer mehr in den Mittelpunkt. So tritt bei Albinus das Beiwerk deutlich in den Hintergrund. In Hallers Icones wird die Staffage oft völlig vernachlässigt. Im Bild wird nicht mehr eine zufällige Variante, sondern die über viele Sektionen ermittelte Norm festgehalten. 


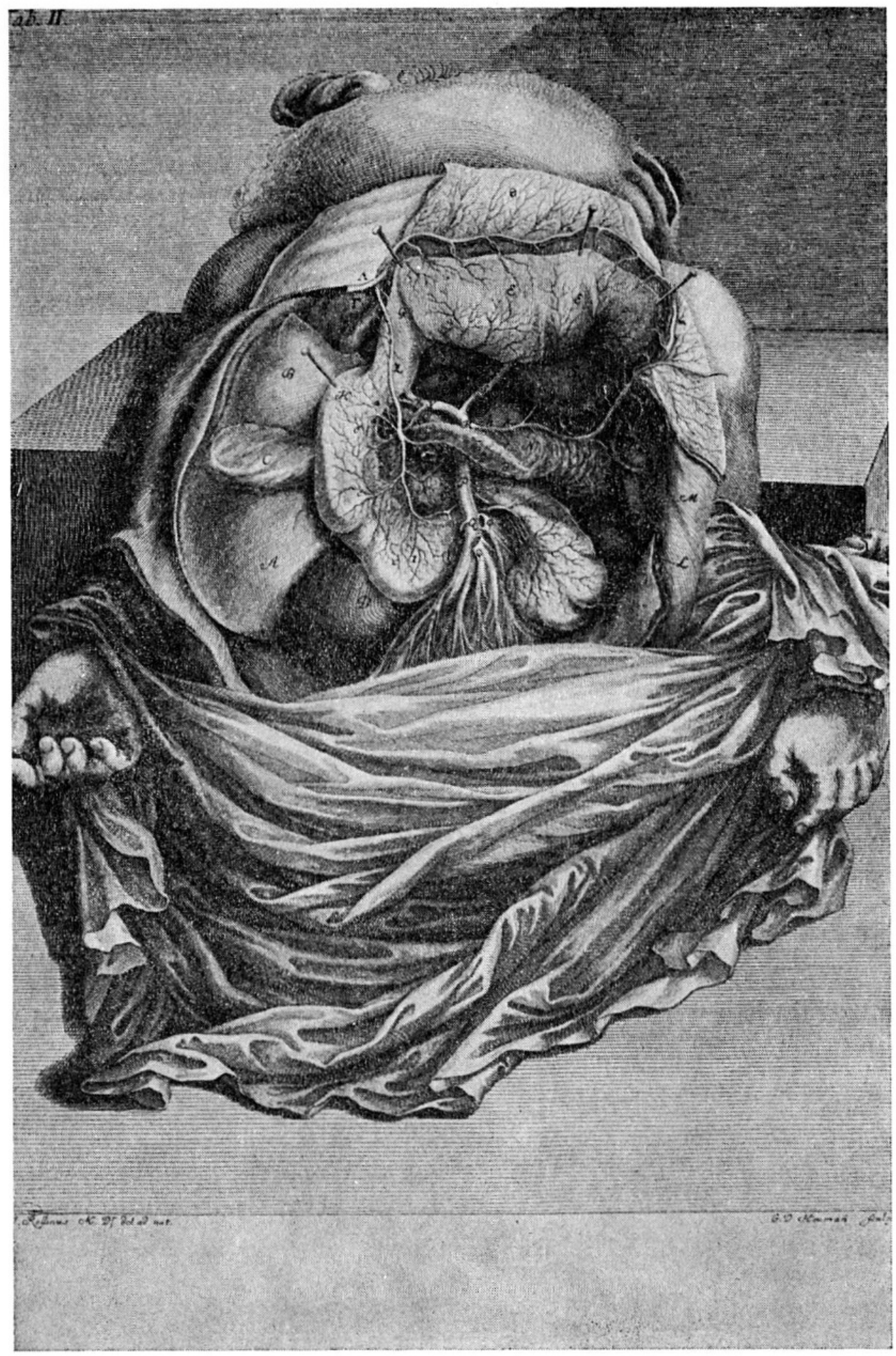

Abb. 3. Albrecht von Haller, Icones anatomicae, Göttingen 1743-1756, Fasc. II, Tab. II. Kupferstich: $32,5 \times 25 \mathrm{~cm}$. Zeichner: Christian Jeremias Rollin (1707 bis nach 1778). Stecher: Georg Daniel Heumann (1691-1759) 
Für den Künstler engt sich dadurch der Raum künstlerischer Entfaltung und Phantasie immer mehr ein. Die anatomische Illustration ist bald nicht mehr ein Zeugnis zeitgenössischer Kunst, sondern wird zum bloßen didaktischen Hilfsmittel des Anatomen. Diese Tendenz, die sich an der Schwelle vom 17. in das 18. Jahrhundert ankündigt, wird im 19. Jahrhundert durch die Einführung der Lithographie weitergeführt und verstärkt.

\section{Danksagung}

Die Arbeit wurde von Herrn Prof. Dr. med. H. M. Koelbing in vielfältiger Weise unterstützt. Meinem Lehrer, Herrn Prof. Dr. med. St. Kubik, danke ich für die Durchsicht des Manuskriptes.

\section{Anmerkungen}

1 Ackerknecht, E. H., Manieristische Anatomie: Frederik Ruysch und seine Sammlung. Image Roche 43 (1971) 27-32.

2 Cetto, A.M., Das Theatrum Anatomicum zu Leiden. CIBA-Symposium 6 (1958) 169 bis 172.

${ }^{3}$ Rohen, J.W., Rembrandts Anatomie des Dr. med. Tulp. Monatsspiegel 1 (1964) 3-7.

4 Faller, A., Die Entwicklung der makroskopisch-anatomischen Präparierkunst von Galen bis zur Neuzeit. Acta anat., suppl. 7 (1948) 1-111 (zitiert: Faller).

5 Wurzbach, A. von, Niederländisches Künstlerlexikon, Bd. 2, Amsterdam: Israël 1963, S. $526 \mathrm{ff}$.

6 Herrlinger, R., Bidloos «Anatomia» - Prototyp barocker Illustration? Gesnerus 23 (1966) 40-47 (zitiert: Herrlinger).

7 Herrlinger, R., Das erste lithographisch illustrierte Lehrbuch der Anatomie. Sudhoffs Archiv 47 (1963) 224-236.

${ }^{8}$ Herrlinger, S. 41 .

9 Herrlinger, S. 42.

10 Faller, S. 84.

11 Herrlinger, S. 43.

12 Choulant, L., Geschichte und Bibliographie der anatomischen Abbildung, Leipzig: Weigel 1852, S. 117 (zitiert: Choulant).

13 Choulant, S.114.

14 Choulant, S. 114-116.

15 Thieme, U., und Becker, F., Allgemeines Lexikon der bildenden Künstler, Bd. 35, Leipzig: Seemann 1942, S. 141.

${ }^{16}$ Soemmerring, S.T., Abbildungen des menschlichen Auges, Frankfurt a. M.: Varrentrapp und Wenner 1801, S. 3.

17 Gloor, B., Die künstlerischen Mitarbeiter an den naturwissenschaftichen und medizinischen Werken Albrecht von Hallers, Bern: Paul Haupt 1958 (zitiert: Gloor).

18 Hyrtl, J., Lehrbuch der Anatomie des Menschen, 15. Aufl., Wien: Braumüller 1881, S. 63.

19 Gloor, S. 19. 


\section{Summary}

During the time between the publication of the "Fabrica" by Vesalines and the middle of the 17th century there was little variation in the style of anatomical illustrations. Casserio's "Tabulae Anatomicae" as well as Ruysch's curiosities are famous examples of the style mentioned. An almost surrealistic manner of engraving dominated this period.

In the second half of the 17 th century we state a marked change in the illustrations. The engravings by Lairesse resemble still lives, representing their objects in a naturalistic manner, taking into account individual variations and traits of character. - The illustrations by Albinus and Haller are strictly limited to their essential purpose; they do not show dispensable accessories any longer.

Contrary to general art which grew more and more imaginative, anatomical engravings were engraved to serve their didactic purpose exclusively.

Hans-Konrad Schmutz

Abteilung für Makroskopie des Anatomischen Institutes

(Leitung: Prof. Dr. med. St. Kubik)

Gloriastraße 19

8006 Zürich 